\title{
SOME INTEGRAL TYPE WEAKLY COMPATIBLE CONTRACTION IN MODULAR METRIC SPACES
}

\author{
Renu Chugh ${ }^{1}$, Naresh Kumar ${ }^{2} \S$ \\ ${ }^{1,2}$ Department of Mathematics \\ M.D. University \\ Rohtak, 124001, Haryana, INDIA
}

\begin{abstract}
In this paper, we prove some fixed point theorems for integral type weakly compatible contraction in modular metric spaces which is more general than a metric. Our results generalize the results of Hossein Rahimpoor et.al., see [19].
\end{abstract}

AMS Subject Classification: 46A80, 47H10

Key Words: modular metric space, weakly compitable maps, coincedence point

\section{Introduction and Preliminaries}

In 1950, [1] Nakano introduced the notion of classical modular spaces and Musilak and Orlicz redefine this notion in 1959 [2, 3]. Jungck [4] generalized the Banach mapping contraction principle by proving the common fixed point theorem for commuting mappings in 1976. Many authors extended and generalized this result in various ways. In 1982, Seesa [5] defined weak commutivity. Further Jungck $[6,7,8]$ introduced the concept of compatibility and weak compatibility mappings, which is generalization of weak commutativity. In [9] Branciari proved a fixed point theorem for single mapping which satisfy a integral type contraction in Banach contraction principle.

Received: $\quad$ September 9, 2016

Revised: December 13, 2016

Published: $\quad$ February 28, 2017

$\S_{\text {Correspondence author }}$ (c) 2017 Academic Publications, Ltd. url: www.acadpubl.eu 
Vijayaraju et. al. [10] extended this result for pair of mappings. Moreover, Rajaniand Moradi[11] prove the common fixed point theorem of integral type in Modular spaces. In 2010, V. V. Chistiyakov [12] introduced the more general concept of modular metric spaces. Later, many authors $[13,14,15,16,17$, $18]$ have extended and generalized the fixed point problems in modular metric spaces.

In this paper, we prove some coincidence and common fixed point theorems for a weakly contractive mappings of integral type in modular metric spaces, Our results generalize and extend the results of hossein Rahimpoor et. al. [19].

Definition 1 (see [12]). A function $w_{\lambda}(x, y)=w(\lambda, x, y):(0, \infty) \times X \times$ $X \longrightarrow[0, \infty]$ is said to be a metric modular on a non empty set $X$ if it satisfies the following three axioms:

(i) given $x, y \in X, w_{\lambda}(x, y)=0$ for all $\lambda>0$ if and only if $x=y$;

(ii) $w_{\lambda}(x, y)=w_{\lambda}(y, x)$ for all $\lambda>0$ and $x, y \in X$;

(iii) $w_{\lambda+\mu}(x, y) \leq w_{\lambda}(x, z)+w_{\mu}(z, y)$ for all $\lambda, \mu>0$ and $x, y, z \in X$. if instead of $(i)$ we have only the condition;

$(i)^{\prime} w_{\lambda}(x, x)=0$ for all $\lambda>0$ and $x \in X$, then $w$ is said to be a metric pseudo -modular on $X$. And if $w$ satisfy $(i)^{\prime}$ and;

(ii) ${ }^{\prime}$ given $x, y \in X$, if there exists $\lambda>0$, possibly depending on $x$ and $y$ such that $w_{\lambda}(x, y)=0$ then $x=y$, with the condition $w$ is called a convex modular on $X$. if instead of (iii) we replace the following condition:

$(i i i)^{\prime} w_{\lambda+\mu}(x, y) \leq \frac{\lambda}{\lambda+\mu} w_{\lambda}(x, z)+\frac{\mu}{\lambda+\mu} w_{\lambda}(z, y)$ for all $\lambda, \mu>0$ and $x, y, z \in$ $X$; then $w$ is called a convex modular on $X$.

Example 2 (see [20]). Let $\lambda>0$ and $x, y \in X$, then the following are simple examples of metric ( pseudo) modulars on a set $X$.

(a) $w_{\lambda}(x, y)=\infty$ if $x \neq y, w_{\lambda}(x, y)=0$ if $x=y$ and if $(X, d)$ is a (pseudo) metric space with (pseudo) metric $d$ then we also have:

(b) $w_{\lambda}(x, y)=\frac{d(x, y)}{\phi(\lambda)}$, where $\phi:(0, \infty) \rightarrow(0, \infty)$ is a non decreasing function:

(c) $w_{\lambda}(x, y)=\infty$ if $\lambda \leq d(x, y)$ and $w_{\lambda}(x, y)=0$ if $\lambda>d(x, y)$;

(d) $w_{\lambda}(x, y)=\infty$ if $\lambda<d(x, y)$ and $w_{\lambda}(x, y)=0$ if $\lambda \geq d(x, y)$;

Remark 3 (see [19]). Let $w$ be a metric modular on a non empty set $X$ for given $x, y \in X$, the function $0<\lambda \longmapsto w_{\lambda}(x, y) \in[0, \infty]$ is non increasing on $(0, \infty)$. In fact, if $0<\lambda<\mu$, then $(i i i),(i)^{\prime}$ and (ii) of above definition 1 implies that

$$
w_{\mu}(x, y) \leq w_{\mu-\lambda}(x, x)+w_{\lambda}(x, y)=w_{\lambda}(x, y) \text { for all } x, y \in X
$$


We conclude from above definition that if $x_{0} \in X$, the set $X_{w}=\{x \in X$ : $\left.\lim _{\lambda \rightarrow \infty} w_{\lambda}\left(x, x_{0}\right)=0\right\}$ is a metric space, called a modular space, whose metric is given by $d_{w}(x, y)=\inf \left\{\lambda>0: w_{\lambda}(x, y) \leq \lambda, x, y \in X_{w}\right\}$ and the modular set $X_{w}$ is a metric space, see theorem 2.6 of [21]. we also put $X_{w}^{*} \equiv X_{w}^{*}\left(x_{0}\right)=$ $\left\{x \in X: \exists \lambda=\lambda(x)>0\right.$ such that $\left.w_{\lambda}\left(x, x_{0}\right)<\infty\right\}$. and also we observe that from [21] that if $X$ is a real linear space, $\rho: X \rightarrow[0, \infty]$ and $w_{\lambda}(x, y)=\rho\left(\frac{x-y}{\lambda}\right)$ for all $\lambda>0$ and $x, y \in X$, then $\rho$ is a modular on $X$ if and only if $w$ is metric modular on $X$.

Definition 4. [20] let $X_{w}$ be a modular metric space.

(a) the sequence $\left\{x_{n}\right\}_{n=1}^{\infty}$ in $X_{w}$ is said to be modular convergent ( $w-$ convergent) to $x \in X$ if there exists a $\lambda>0$, possibly depend on $x_{n}$ and $x$, such that $w_{\lambda}\left(x_{n}, x\right) \rightarrow 0$ as $n \rightarrow \infty$ for all $\lambda>0$.

(b) The sequence $\left\{x_{n}\right\}_{n=1}^{\infty}$ in $X_{w}$ is said to be modular Cauchy (w-Caushy) to $x \in X$ if there exists a $\lambda>0$ such that $w_{\lambda}\left(x_{n}, x_{m}\right) \rightarrow 0$ as $m, n \rightarrow \infty$ for all $\lambda>0$ i. e. for all $\epsilon>0$ there exists $n_{0}(\epsilon) \in N$ such that for all $n, m \geq n_{0}(\epsilon) ; w_{\lambda}\left(x_{n}, x_{m}\right) \leq \epsilon$.

(c) The modular metric space $X_{w}$ is said to be complete $(w-$ complete) if each modular Cauchy sequence from $X_{w}$ is modular convergent in $X_{w}$. i. e. if $x_{n} \subset X_{w}$ and there exists $\lambda>o$ such that $w_{\lambda}\left(x_{n}, x_{m}\right) \rightarrow 0$ as $m, n \rightarrow \infty$ then there exists $x \in X_{w}$ such that $w_{\lambda}\left(x_{n}, x\right) \rightarrow 0$.

(d) [14] A modular $w$ on $X$ is said to satisfy the $\triangle_{2}$-condition if for a sequence $\left\{x_{n}\right\} \subset X_{w}$ and $x \in X_{w}$, there exists a $\lambda>0$, possibly depending on $\left\{x_{n}\right\}$ and $x$, such that $w_{\lambda}\left(x_{n}, x\right) \rightarrow 0$ as $n \rightarrow \infty$ for all $\lambda>0$.

Remark 5 (see [12]). Let $X_{w}$ be a modular metric spaces, then the metric convergent (with respect $d_{w}$ ) implies the modular convergent i. e. $d_{w}\left(x_{n}, x\right) \rightarrow$ 0 as $n \rightarrow \infty$ if and only if $w_{\lambda}\left(x_{n}, x\right) \rightarrow 0$ as $n \rightarrow \infty$ for all $\lambda>0$.

Definition 6 (see [19]). let $w$ be a metric modular on a non empty set $X$ and $S$ and $T$ are self maps on $X_{w}$. A point $x \in X_{w}$ is called a coincedence point of $S$ and $T$ if $T x=S x$. The mappings $S$ and $T$ are said to be weakly compatible if they commute at their coincedence point i. e. $T S x=S T x$ Whenever $T x=S x$.

Suppose $T\left(X_{w}\right) \subset S\left(X_{w}\right)$. Let $x_{0}$ be an arbitrary point in $X_{w}$. Since $T\left(X_{w}\right) \subseteq S\left(X_{w}\right)$ there exist a point $x_{1} \in X_{w}$ such that $T x_{0}=S x_{1}$. By repeating this process we construct the sequence $\left\{x_{n}\right\}$ in $X_{w}$ such that $S x_{n}=T x_{n-1}$ for all $n \geq 1$, we say that $\left\{T x_{n}\right\}$ is a $T-S-$ sequence with initial point $x_{0}$. 


\section{Main Results}

Theorem 7. Let $X_{w}$ be a modular metric space and $S, T: X_{w} \rightarrow X_{w}$ be two mappings such that $T\left(X_{w}\right) \subseteq S\left(X_{w}\right)$ be a ${ }^{W}$-complete subspaces of $X_{w}$. Suppose there exits numbers $\alpha, \beta, \gamma \in[0,1)$ such that the following assertion for all $x, y \in X_{w}$ and $\lambda>0$ hold:

1. $(\alpha+2 \beta+2 \gamma)<1$ for all $0 \leq \alpha, \beta, \gamma<1$;

2. We have

$$
\begin{aligned}
\int_{0}^{w_{\lambda}(T x, T y)} \phi(t) d t & \leq \alpha \int_{0}^{w_{\lambda}(S x, S y)} \phi(t) d t \\
+\beta\left[\int_{0}^{w_{\lambda}(S x, T x)} \phi(t) d t+\int_{0}^{w_{\lambda}(S y, T y)} \phi(t) d t\right] & +\gamma\left[\int_{0}^{w_{2 \lambda}(S x, T y)} \phi(t) d t+\int_{0}^{w_{\lambda}(S y, T x)} \phi(t) d t\right],
\end{aligned}
$$

where $\phi: R^{+} \longrightarrow R^{+}$is a Lebesgue integral mapping which is summable, non-negative and for all

$$
\epsilon>0, \quad \int_{0}^{\epsilon} \phi(t) d t>0
$$

3. If $\int_{0}^{w_{\lambda}(S x, T y)} \phi(t) d t<\infty$, then $T$ and $S$ have a coincidence point.

Proof. Let $x_{0}$ be an arbitrary point in $X_{w}$. Since $T\left(X_{w}\right) \subseteq S\left(X_{w}\right)$ there exist a T-S sequence $\left\{T x_{n}\right\}$ in $X_{w}$ such that $S x_{n}=T x_{n-1}$ for all $n \geq 1$. Now we take $x=x_{n}$ and $y=x_{n+1}$ in (2), we get

$$
\begin{aligned}
& \int_{0}^{w_{\lambda}\left(T x_{n}, T x_{n+1}\right)} \phi(t) d t \\
& \leq \alpha \int_{0}^{w_{\lambda}\left(S x_{n}, S x_{n+1}\right)} \phi(t) d t+\beta\left[\int_{0}^{w_{\lambda}\left(S x_{n 1}, S x_{n+1}\right)} \phi(t) d t+\int_{0}^{w_{\lambda}\left(T x_{n}, T x_{n+1}\right)} \phi(t) d t\right] \\
& \quad+\gamma\left[\int_{0}^{w_{2 \lambda}\left(T x_{n-1}, T x_{n+1}\right)} \phi(t) d t+\int_{0}^{w_{\lambda}\left(S x_{n+1}, S x_{n+1}\right)} \phi(t) d t\right],
\end{aligned}
$$

for all $\lambda>0$.

On the other hand

$$
\int_{0}^{w_{2 \lambda}\left(T x_{n-1}, T x_{n-1}\right)} \phi(t) d t \leq \int_{0}^{w_{\lambda}\left(T x_{n-1}, T x_{n}\right)} \phi(t) d t+\int_{0}^{w_{\lambda}\left(T x_{n}, T x_{n+1}\right)} \phi(t) d t
$$




$$
=\int_{0}^{w_{\lambda}\left(S x_{n}, S x_{n+1}\right)} \phi(t) d t+\int_{0}^{w_{\lambda}\left(T x_{n}, T x_{n+1}\right)} \phi(t) d t
$$

So we obtain

$$
\begin{aligned}
& \int_{0}^{w_{\lambda}\left(T x_{n}, T x_{n+1}\right)} \phi(t) d t \\
& \leq \lambda \int_{0}^{w_{\lambda}\left(S x_{n}, S x_{n+1}\right)} \phi(t) d t+\beta\left[\int_{0}^{w_{\lambda}\left(S x_{n}, S x_{n+1}\right)} \phi(t) d t+\int_{0}^{w_{\lambda}\left(T x_{n}, T x_{n+1}\right)} \phi(t) d t\right] \\
& \quad+\gamma\left[\int_{0}^{w_{\lambda}\left(T x_{n}, T x_{n+1}\right)} \phi(t) d t+\int_{0}^{w_{\lambda}\left(S x_{n}, S x_{n+1}\right)} \phi(t) d t\right] .
\end{aligned}
$$

this implies that

$$
\int_{0}^{w_{\lambda}\left(T x_{n}, T x_{n+1}\right)} \phi(t) d t \leq k \int_{0}^{w_{\lambda}\left(S x_{n}, S x_{n+1}\right)} \phi(t) d t \text { for all } n \in N
$$

where $k=\frac{\alpha+\beta+\gamma}{1-\beta-\gamma}<1$. So by induction we get

$$
\int_{0}^{w_{\lambda}\left(T x_{n}, T x_{n+1}\right)} \phi(t) d t \leq k^{n} \int_{0}^{w_{\lambda}\left(T x_{0}, T x_{1}\right)} \phi(t) d t \text { for all } n \in N .
$$

By (2) in a straightforward way, we implies that $\left\{T x_{n}\right\}$ is a $w-$ Cauchy sequence. Since $S\left(X_{w}\right)$ is a $w$-complete, there exists $u, v \in X_{w}$ such that $u=S(v)$ and $T x_{n} \underset{w}{\rightarrow} u$ as $n \rightarrow \infty$. Since $w$ satisfy in the $\Delta_{2}$-condition on $X$ we get

$$
\lim _{n \rightarrow \infty} \int_{0}^{w_{\lambda}\left(T x_{n}, u\right)} \phi(t) d t=0 \text { for all } \lambda>0 .
$$

Therefore

$$
\lim _{n \rightarrow \infty} \int_{0}^{w_{\lambda}\left(T x_{n}, u\right)} \phi(t) d t=\int_{0}^{w_{\lambda}\left(S x_{n}, u\right)} \phi(t) d t=0 \text { for all } \lambda>0 .
$$

Now by taking $x=x_{n}$ and $y=v$ in (2), we obtain that

$$
\begin{aligned}
\int_{0}^{w_{\lambda}\left(T x_{n}, T v\right)} \phi(t) d t & \leq \alpha \int_{0}^{w_{\lambda}\left(S x_{n}, S v\right)} \phi(t) d t \\
+\beta\left[\int_{0}^{w_{\lambda}\left(S x_{n}, T x_{n}\right)} \phi(t) d t+\int_{0}^{w_{\lambda}(S v, T v)} \phi(t) d t\right] & \\
& +\gamma\left[\int_{0}^{w_{2 \lambda}\left(S x_{n}, T v\right)} \phi(t) d t+\int_{0}^{w_{\lambda}\left(S v, T x_{n}\right)} \phi(t) d t\right] .
\end{aligned}
$$


By Remark 3 the function $\lambda \mapsto w_{\lambda}(x, y)$ is non -increasing, so we have

$$
\begin{gathered}
\int_{0}^{w_{\lambda}\left(T x_{n}, T v\right)} \phi(t) d t \leq \alpha \int_{0}^{w_{\lambda}\left(S x_{n}, S v\right)} \phi(t) d t \\
+\beta\left[\int_{0}^{w_{\lambda}\left(S x_{n}, T x_{n}\right)} \phi(t) d t+\int_{0}^{w_{\lambda}(S v, T v)} \phi(t) d t\right] \\
+\gamma\left[\int_{0}^{w_{\lambda}\left(S x_{n}, T x_{n}\right)} \phi(t) d t+\int_{0}^{w_{\lambda}\left(T x_{n}, T v\right)} \phi(t) d t+\int_{0}^{w_{\lambda}\left(S v, T x_{n}\right)} \phi(t) d t\right] .
\end{gathered}
$$

Using (3) and letting $n \rightarrow \infty$ in the above inequality, we get

$$
\begin{aligned}
& \int_{0}^{w_{\lambda}(S v, T v)} \phi(t) d t \leq \alpha \int_{0}^{w_{\lambda}(S v, S v)} \phi(t) d t \\
&+\beta\left[\int_{0}^{w_{\lambda}(S v, T v)} \phi(t) d t+\int_{0}^{w_{\lambda}(S v, T v)} \phi(t) d t\right] \\
&+\gamma\left[\int_{0}^{w_{\lambda}(S v, T v)} \phi(t) d t+\int_{0}^{w_{\lambda}(S v, T v)} \phi(t) d t+\int_{0}^{w_{\lambda}(S v, S v)} \phi(t) d t\right] .
\end{aligned}
$$

So, $1-2 \beta-2 \gamma \int_{0}^{w_{\lambda}(S v, T v)} \phi(t) d t \leq 0$ for all $\lambda>0$, hence $S v=T v=u$. Thus we have proved that $S$ and $T$ have a coincidence point.

Remark 8. If we take $\phi(t)=1$ in above theorem then we get Theorem 10 of [19].

Theorem 9. In addition to the hypothesis of theorem 10, of [19] suppose that $T$ and $S$ are weakly compatible, then $T$ and $S$ have a unique common fixed point. Further, for any $x_{0} \in X_{w}$, the $T-S$-sequence $\left\{T x_{n}\right\}$ with initial point $x_{0}$ modular converges to the common fixed point.

Proof. Assume that $S, T$ are weakly compatible then $S u=S T v=T S v=$ $T u$, Now we want to show that $T u=u=T v$. Suppose $\int_{0}^{w_{\lambda}(T u, T v)} \phi(t) d t>0$ for all $\lambda>0$, by taking $x=u$ and $y=v$ in (2) we get

$$
\begin{aligned}
\int_{0}^{w_{\lambda}(T u, T v)} \phi(t) d t & \leq \alpha \int_{0}^{w_{\lambda}(S u, S v)} \phi(t) d t \\
+\beta\left[\int_{0}^{w_{\lambda}(S u, T u)} \phi(t) d t+\int_{0}^{w_{\lambda}(S v, T v)} \phi(t) d t\right] & +\gamma\left[\int_{0}^{w_{2 \lambda}(S u, T v)} \phi(t) d t+\int_{0}^{w_{\lambda}(S v, T u)} \phi(t) d t\right],
\end{aligned}
$$


for all $u, v \in X_{w}$ and $\lambda>0$, i.e.

$$
\begin{aligned}
\int_{0}^{w_{\lambda}(T u, T v)} \phi(t) d t & \leq \alpha \int_{0}^{w_{\lambda}(T u, T v)} \phi(t) d t \\
+\beta\left[\int_{0}^{w_{\lambda}(T u, T u)} \phi(t) d t+\int_{0}^{w_{\lambda}(T v, T v)} \phi(t) d t\right] & +\gamma\left[\int_{0}^{w_{2 \lambda}(T u, T v)} \phi(t) d t+\int_{0}^{w_{\lambda}(T v, T u)} \phi(t) d t\right] .
\end{aligned}
$$

By Remark 2 we have

$$
\begin{aligned}
& \int_{0}^{w_{\lambda}(T u, T v)} \phi(t) d t \\
& \leq \alpha \int_{0}^{w_{\lambda}(T u, T v)} \phi(t) d t+\gamma\left[\int_{0}^{w_{\lambda}(T u, T v)} \phi(t) d t+\int_{0}^{w_{\lambda}(T v, T u)} \phi(t) d t\right],
\end{aligned}
$$

for all $\lambda>0$. This implies that $1-\alpha-2 \gamma \int_{0}^{w_{\lambda}(T u, T v)} \phi(t) d t \leq 0$. which is a contradiction by assumption. Therefore $S u=T u=T v=u$, and hence $S, T$ have a common fixed point. For the uniqueness of common fixed point, Suppose that $u$ and $z$ be two common fixed points i. e $T u=S u=u$ and $T z=S z=z$.

Taking $x=u$ and $y=z$ in (2), we obtain that

$$
\begin{aligned}
\int_{0}^{w_{\lambda}(T u, T z)} \phi(t) d t & \leq \alpha \int_{0}^{w_{\lambda}(S u, S z)} \phi(t) d t \\
+ & \beta\left[\int_{0}^{w_{\lambda}(S u, T u)} \phi(t) d t+\int_{0}^{w_{\lambda}(S z, T z)} \phi(t) d t\right] \\
& +\gamma\left[\int_{0}^{w_{2 \lambda}(S u, T z)} \phi(t) d t+\int_{0}^{w_{\lambda}(S z, T u)} \phi(t) d t\right],
\end{aligned}
$$

for all $\lambda>0$.

So $(1-\alpha-2 \gamma) \int_{0}^{w_{\lambda}(T u, T z)} \phi(t) d t \leq 0$ for all $\lambda>0$, which is a contraction. Therefore $\int_{0}^{w_{\lambda}(u, z)} \phi(t) d t=0$ for all $\lambda>0$ and so $u=z$. Clearly, for any $x_{0} \in X_{w}$, the T-S-sequence $\left\{T x_{n}\right\}$ with initial point $x_{0}$ converges to the unique common fixed point.

Remark 10. By putting $\phi(t)=1$ in above theorem then it reduces the theorem 11 of [19]. By setting $S=I X_{w}$, we deduce the following result of fixed point for one self mapping from theorem 10 of [19]. 
Corollary 11. Let $X_{w}$ be a $w$-complete modular metric space and $T: X_{w} \rightarrow X_{w}$, such that for all $\lambda>0$ and $x, y \in X_{w}, \int_{0}^{w_{\lambda}(x, T y)} \phi(t) d t<\infty$ and $\int_{0}^{w_{\lambda}(T x, T y)} \phi(t) d t \leq \alpha \int_{0}^{w_{\lambda}(x, y)} \phi(t) d t+\beta\left[\int_{0}^{w_{\lambda}(x, T x)} \phi(t) d t+\int_{0}^{w_{\lambda}(y, T y)} \phi(t) d t\right]+$ $\gamma\left[\int_{0}^{w_{2 \lambda}(x, T y)} \phi(t) d t+\int_{0}^{w_{\lambda}(y, T x)} \phi(t) d t\right]$, where $(\alpha+2 \beta+2 \gamma)<1,0 \leq \alpha, \beta, \gamma<1$. and $\phi: R^{+} \longrightarrow R^{+}$is a Lebesgue integral mapping which is summable, nonnegative and for all $\epsilon>0, \int_{0}^{\epsilon} \phi(t) d t>0$. Then $T$ has a unique fixed point. Further, for any $x_{0} \in X_{w}$, the picard sequence $\left\{T x_{n}\right\}$ with initial point $x_{0}$ modular converges to the fixed point.

Corollary 12. Let $X_{w}$ be a $w$-complete modular metric space and $T: X_{w} \rightarrow X_{w}$, such that for all $\lambda>0$ and $x, y \in X_{w}, \int_{0}^{w_{\lambda}(x, T y)} \phi(t) d t<\infty$ and $\int_{0}^{w_{\lambda}(T x, T y)} \phi(t) d t \leq \alpha \int_{0}^{w_{\lambda}(x, y)} \phi(t) d t$ where $0 \leq \alpha<1$ and $\phi: R^{+} \longrightarrow R^{+}$ is a Lebesgue integral mapping which is summable, non-negative and for all $\epsilon>0, \int_{0}^{\epsilon} \phi(t) d t>0$. Then $T$ has a unique fixed point.

Corollary 13. Let $X_{w}$ be a $w$-complete modular metric space and $T: X_{w} \rightarrow X_{w}$, such that for all $\lambda>0$ and $x, y \in X_{w}, \int_{0}^{w_{\lambda}(x, T y)} \phi(t) d t<\infty$ and $\int_{0}^{w_{\lambda}(T x, T y)} \phi(t) d t \leq \beta\left[\int_{0}^{w_{\lambda}(x, T x)} \phi(t) d t+\int_{0}^{w_{\lambda}(y, T y)} \phi(t) d t\right]$ where $\beta \in\left[0, \frac{1}{2}\right)$ and $\phi: R^{+} \rightarrow R^{+}$is a Lebesgue integral mapping which is summable, non-negative and for $\epsilon>0, \int_{0}^{t} \phi(t) d t>0$. then $T$ has a unique fixed point.

Theorem 14. Let $X_{w}$ be a modular metric space and $S, T: X_{w} \longrightarrow X_{w}$ be two mappings such that $T\left(X_{w}\right) \subseteq S\left(X_{w}\right)$ be a $w$-complete subspace of $X_{w}$. Suppose there exists mappings $\alpha, \beta, \gamma, \mu: X_{w} \longrightarrow[0,1)$ such that the following assertions for all $x, y \in X_{w}$. and $\lambda>0$ holds:

1. $\alpha(T x) \leq \alpha(S x), \beta(T x) \leq \beta(S x), \gamma(T x) \leq \gamma(S x), \mu(T x) \leq \mu(S x)$;

2. $(\alpha+2 \beta+\gamma+\mu)<1$ for all $0 \leq \alpha, \beta, \gamma, \mu<1$;

3. $\int_{0}^{w_{\lambda}(T x, T y)} \phi(t) d t \leq \alpha(S x) \int_{0}^{w_{\lambda}(S x, S y)} \phi(t) d t+\beta(S x) \int_{0}^{w_{2 \lambda}(S x, T y)} \phi(t) d t+$ $\gamma(S x) \int_{0}^{w_{\lambda}(S x, T x)} \phi(t) d t+\mu(S x) \int_{0}^{w_{\lambda}(S y, T y)} \phi(t) d t$

4. $\int_{0}^{w_{\lambda}(S x, T y)} \phi(t) d t<\infty$ where $\phi: R^{+} \longrightarrow R^{+}$is a Lebesgue integrable mapping which is summable, non negative and for all $\epsilon>0 \int_{0}^{\epsilon} \phi(t) d t>0$. Then $T$ and $S$ have a unique common fixed point.

Proof. Let $x_{0}$ be an arbitrary point in $X_{w}$. Since $T\left(X_{w}\right) \subseteq S\left(X_{w}\right)$ there exist a $T-S-$ sequence $\left\{T x_{n}\right\}$ in $X_{w}$ such that $S x_{n}=T x_{n-1}$ for all $n \geq 1$. .... (4) 
From (1), (3) and (4) we have

$$
\begin{aligned}
& \int_{0}^{w_{\lambda}\left(S x_{n}, S x_{n+1}\right)} \phi(t) d t=\int_{0}^{w_{\lambda}\left(T x_{n-1}, T x_{n}\right)} \phi(t) d t \\
& \leq \alpha\left(S x_{n-1}\right) \int_{0}^{w_{\lambda}\left(S x_{n-1}, S x_{n}\right)} \phi(t) d t+\beta\left(S x_{n-1}\right) \int_{0}^{w_{2 \lambda}\left(S x_{n-1}, T x_{n}\right)} \phi(t) d t \\
& +\gamma\left(S x_{n-1}\right) \int_{0}^{w_{\lambda}\left(S x_{n-1}, T x_{n-1}\right)} \phi(t) d t+\mu\left(S x_{n-1}\right) \int_{0}^{w_{\lambda}\left(S x_{n}, T x_{n}\right)} \phi(t) d t \\
& =\alpha\left(T x_{n-2}\right) \int_{0}^{w_{\lambda}\left(S x_{n-1}, S x_{n}\right)} \phi(t) d t+\beta\left(T x_{n-2}\right) \int_{0}^{w_{2 \lambda}\left(S x_{n-1}, S x_{n+1}\right)} \phi(t) d t \\
& +\gamma\left(T x_{n-2}\right) \int_{0}^{w_{\lambda}\left(S x_{n-1}, S x_{n}\right)} \phi(t) d t+\mu\left(T x_{n-2}\right) \int_{0}^{w_{\lambda}\left(S x_{n}, S x_{n+1}\right)} \phi(t) d t \\
& \leq \alpha\left(S x_{n-2}\right) \int_{0}^{w_{\lambda}\left(S x_{n-1}, S x_{n}\right)} \phi(t) d t+\beta\left(S x_{n-2}\right) \int_{0}^{w_{2 \lambda}\left(S x_{n-1}, S x_{n+1}\right)} \phi(t) d t \\
& +\gamma\left(S x_{n-2}\right) \int_{0}^{w_{\lambda}\left(S x_{n-1}, S x_{n}\right)} \phi(t) d t+\mu\left(S x_{n-2}\right) \int_{0}^{w_{\lambda}\left(S x_{n}, S x_{n+1}\right)} \phi(t) d t \\
& \leq \alpha\left(S x_{0}\right) \int_{0}^{w_{\lambda}\left(S x_{n-1}, S x_{n}\right)} \phi(t) d t+\beta\left(S x_{0}\right) \int_{0}^{w_{2 \lambda}\left(S x_{n-1}, S x_{n+1}\right)} \phi(t) d t \\
& +\gamma\left(S x_{0}\right) \int_{0}^{w_{\lambda}\left(S x_{n-1}, S x_{n}\right)} \phi(t) d t+\mu\left(S x_{0}\right) \int_{0}^{w_{\lambda}\left(S x_{n}, S x_{n+1}\right)} \phi(t) d t \\
& \leq \alpha\left(S x_{0}\right) \int_{0}^{w_{\lambda}\left(S x_{n-1}, S x_{n}\right)} \phi(t) d t+\beta\left(S x_{0}\right)\left[\int_{0}^{w_{\lambda}\left(S x_{n-1}, S x_{n}\right)} \phi(t) d t\right. \\
& \left.+\int_{0}^{w_{\lambda}\left(S x_{n}, S x_{n+1}\right)} \phi(t) d t\right]+\gamma\left(S x_{0}\right) \int_{0}^{w_{\lambda}\left(S x_{n-1}, S x_{n}\right)} \phi(t) d t \\
& +\mu\left(S x_{0}\right) \int_{0}^{w_{\lambda}\left(S x_{n}, S x_{n+1}\right)} \phi(t) d t .
\end{aligned}
$$

This implies that

$$
\int_{0}^{w_{\lambda}\left(S x_{n}, S x_{n+1}\right)} \phi(t) d t \leq\left(\frac{\alpha\left(S x_{0}\right)+\beta\left(S x_{0}\right)+\gamma\left(S x_{0}\right)}{1-\beta\left(S x_{0}\right)-\mu\left(S x_{0}\right)}\right) \int_{0}^{w_{\lambda}\left(S x_{n-1}, S x_{n}\right)} \phi(t) d t
$$

for all $n \geq 1$.

Now we let

$$
k=\frac{\alpha\left(S x_{0}\right)+\beta\left(S x_{0}\right)+\gamma\left(S x_{0}\right)}{1-\beta\left(S x_{0}\right)-\mu\left(S x_{0}\right)}<1 .
$$


Repeating in (5), we get

$$
\int_{0}^{w_{\lambda}\left(S x_{n}, S x_{n+1}\right)} \phi(t) d t+\leq k^{n} \int_{0}^{w_{\lambda}\left(S x_{1}, S x_{0}\right)} \phi(t) d t .
$$

Now, for $m>n \geq 1$, it follows from (6) that

$$
\begin{aligned}
& \int_{0}^{w_{\lambda}\left(S x_{n}, S x_{m}\right)} \phi(t) d t \leq \int_{0}^{w} \frac{\lambda}{m-n}\left(S x_{n}, S x_{n+1}\right) \phi(t) d t \\
& +\int_{0}^{w} \frac{\lambda}{m-n}\left(S x_{n+1}, S x_{n+2}\right) \phi(t) d t+\ldots \\
& +\int_{0}^{w} \frac{\lambda}{m-n}\left(S x_{m-1}, S x_{m}\right) \phi(t) d t
\end{aligned}
$$

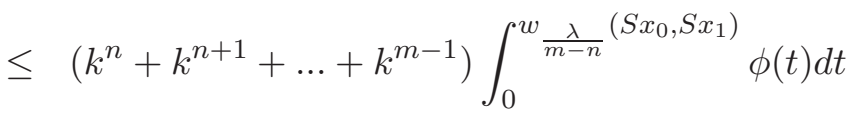

$$
\begin{aligned}
& =\frac{k^{n}-k^{m}}{1-k} \int_{0}^{w} \frac{\lambda}{m-n}{ }^{\left(S x_{0}, S x_{1}\right)} \phi(t) d t .
\end{aligned}
$$

Since $0 \leq k<1$, we conclude that $\left\{S x_{n}\right\}$ is a $w-$ Cauchy sequence in $S\left(X_{w}\right)$, on the other hand by hypothesis $S\left(X_{w}\right)$ is a $w$-complete subspace of $X_{w}$, therefore there exists a point $u \in S\left(X_{w}\right)$ such that $S x_{n} \underset{w}{\rightarrow} u$ as $n \rightarrow \infty$. By hypothesis $w$ satisfy in $\Delta_{2}$ - condition on $X_{w}$, so $\lim _{n \rightarrow \infty} \int_{0}^{w_{\lambda}\left(S x_{n}, u\right)} \phi(t) d t=0$ for all $\lambda>0$.

Now, we claim that $u$ is a common fixed point. Suppose that $T u \neq u$ or $S u \neq u$. Then we have

$$
\begin{aligned}
0 & <\inf \left\{\int_{0}^{w_{\lambda}(T x, u)} \phi(t) d t+\int_{0}^{w_{\lambda}(S x, u)} \phi(t) d t+\int_{0}^{w_{\lambda}(T x, S x)} \phi(t) d t: x \in X_{w}\right\} \\
& \leq \inf \left\{\int_{0}^{w_{\lambda}\left(T x_{n}, u\right)} \phi(t) d t+\int_{0}^{w_{\lambda}\left(S x_{n}, u\right)} \phi(t) d t+\int_{0}^{w_{\lambda}\left(T x_{n}, S x_{n}\right)} \phi(t) d t: n \geq 1\right\} \\
& =\inf \left\{\int_{0}^{w_{\lambda}\left(S x_{n+1}, u\right)} \phi(t) d t+\int_{0}^{w_{\lambda}\left(S x_{n}, u\right)} \phi(t) d t+\int_{0}^{w_{\lambda}\left(S x_{n}, S x_{n+1}\right)} \phi(t) d t\right\} \\
& \leq \inf \left\{\int_{0}^{w_{\lambda}\left(S x_{n+1}, u\right)} \phi(t) d t+\int_{0}^{w_{\lambda}\left(S x_{n}, u\right)} \phi(t) d t+\int_{0}^{w_{\frac{\lambda}{2}}\left(S x_{n}, u\right)} \phi(t) d t\right. \\
& \left.+\int_{0}^{w_{\frac{\lambda}{2}}\left(u, S x_{n+1}\right)} \phi(t) d t: n \geq 1\right\} \longrightarrow 0
\end{aligned}
$$


as $n \rightarrow \infty$ for all $\lambda>0$. which is a contraction. Therefore, this implies that $u=S u=T u$.

For the uniqueness of the common fixed point, Suppose $T u=S u=u$ $T z=S z=z$ be two common fixed points then by taking $x=u$ and $y=z$ in (3), we obtain that

$$
\begin{aligned}
\int_{0}^{w_{\lambda}(u, z)} \phi(t) d t & \leq \alpha(u) \int_{0}^{w_{\lambda}(u, z)} \phi(t) d t+\beta(u) \int_{0}^{w_{2 \lambda}(u, z)} \phi(t) d t \\
& +\gamma(u) \int_{0}^{w_{\lambda}(u, u)} \phi(t) d t+\mu(u) \int_{0}^{w_{\lambda}(z, z)} \phi(t) d t \\
& \leq \alpha(u) \int_{0}^{w_{\lambda}(u, z)} \phi(t) d t+\beta(u) \int_{0}^{w_{\lambda}(u, z)} \phi(t) d t
\end{aligned}
$$

This implies that $(1-\alpha-\beta)(u) \int_{0}^{w_{\lambda}(u, z)} \phi(t) d t \leq 0$ which is a contraction by assumption. By taking the mapping $S$ in theorem 14 as $I X_{w}$, where $I X_{w}$ is an identity mapping on $X_{w}$, we have the following corollary.

Corollary 15. Let $X_{w}$ be a $w$-complete modular metric space and $T: X_{w} \rightarrow X_{w}$. Suppose that there exists mappings $\alpha, \beta, \gamma, \mu: X_{w} \rightarrow[0,1)$ such that the following assertion for all $x, y \in X_{w}$ and $\lambda>0$ hold:

1. $\alpha(T x) \leq \alpha(x), \beta(T x) \leq \beta(x), \gamma(T x) \leq \gamma(x), \mu(T x) \leq \mu(x) ;$

2. $(\alpha+2 \beta+\gamma+\mu)<1$ for all $0 \leq \alpha, \beta, \gamma, \mu<1$;

$\begin{aligned} \int_{0}^{w_{\lambda}(T x, T y)} \phi(t) d t \leq \alpha(x) & \int_{0}^{w_{\lambda}(x, y)} \phi(t) d t+\beta(x) \int_{0}^{w_{2 \lambda}(x, T y)} \phi(t) d t \\ & +\gamma(x) \int_{0}^{w_{\lambda}(x, T x)} \phi(t) d t+\mu(x) \int_{0}^{w_{\lambda}(y, T y)} \phi(t) d t ;\end{aligned}$

4. $\int_{0}^{w_{\lambda}(x, T y)} \phi(t) d t<\infty$ where $\phi: R^{+} \longrightarrow R^{+}$is a Lebesgue integrable mapping which is summable, non negative and for all $\epsilon>0 \int_{0}^{\epsilon} \phi(t) d t>0$. Then $T$ has a unique common fixed point.

\section{References}

[1] H. Nakano, Modular Semi-Ordered Spaces, Tokyo, 1950.

[2] J. Musilak, W. Orlicz, On modular spaces, Studia Mathematica, 18 (1959), 49-65.

[3] J. Musilak, Orlicz Spaces and Modular Spaces, Lecture notes in Maths, vol 1034, Springer, Berlin, 1983.

[4] Jungck, Commuting mappings and fixed point, Am. Math. Mon. , 83 (1976), 261-263. 
[5] Sessa, On a weak commutativity conditions of mappings in fixed point consideration, Publ. Inst. Math. Belgr., 32 (1982), 146-153.

[6] G. Jungck, Compatible mappings and common fixedpoints, Int. J. Math. Sci. , 11 (1986), 771-779.

[7] G. Jungck, Common fixed points for non continuous nonself maps on non-metric space, Far East J. Math. Sci. , 4 (1996), 19-215.

[8] G. Jungck, B. E. Rhoades, Fixed point for set valued functions without continuity, Indian J. Pure App. Math. , 29, No. 3 (1998), 227-238.

[9] Branciari, A fixed point theorem for mapping satisfy a general contractive condition of integral type, Int. J. Math. Sci., 29 (2002), 531-536.

[10] Vijayaraju, P. Rhoades, B. E. Mohanra, A fixed point theorem for mappings satisfying a general contractive condition of integral type, Int. J. Math. Sci. , 15 (2005), 2359-2364.

[11] Razani, A, Moradi, Common fixed point theorems of integral type in integral type in modular spaces, Bull. Iran. Math. Soc., 35 (2009), 11-24.

[12] V. Chistyakov, Modular metric spaces, 1: Basic conspect, Nonlinear Analysis, 72 (2010), 1-14, doi: 10. 1016/j. na. 2009. 04. 057.

[13] P. Chaipunya, C. Mongkolkeha, W. Sintunavarat, P. Kuman, Fixed point theorems for multivalued mappings in modular metric space, Abstract and Applied Analysis, 20012, Article ID 503504, 14 pages, doi: 10. 1155/2012/503504.

[14] V. Chistykov, A fixed point theorem for contractions in modular metric spaces, Preprint submited to Arxiv (2011).

[15] Y. Cho, R. Saadati, G. Sadeghi, Quasi-contraction mappingsin modular metric spaces, Journal of Applied Mathematics (2012), Article I. D. 907951, 5 pages, doi: 10. $1155 / 2012 / 907951$.

[16] C. Mongkolkeha, W. Sintanavarat, P. Kumam, Fixed point theorems for contraction mappings in modular spaces, Fixed Point Theory and Application, 93 (2011), doi: 10. 1186/1687-1812-2011-93.

[17] H. Rahimpoor, A. Ebardian, M. Eshaghi Gordji, A. Johri, Fixed point theory for generalized quasi -contraction maps in modular metric spaces, Journal of Mathematics and Computer Science, 10 (2014), 54-60.

[18] H. Rahimpoor, A. Ebardian, M. Eshaghi gordji, A. Zohri, Some fixed point theorems on modular metric spaces, Acta Universitatis Apulensis, 37 (2014), 161-170.

[19] Hossein Rahimpoor, Ali Ebadian, Madjid Eshaghi Gordji, Ali Zohri, common fixed point theorems in modular metric spaces, International Journal of Pure and Applied Mathematics, 99, No. 3 (2015), 373-383.

[20] Bahareh Azadifar, Ghadir Sadeghi, Reza Saadati, and Choonkil Park, Integral type contraction in modular metric spaces, Journal of Inequalities and App. , 483 (2013), $2-14$. 